\title{
Exploring multilayered collaboration designs
}

\author{
Nancy Law ${ }^{1} \cdot$ Sanna Järvelä ${ }^{2} \cdot$ Carolyn Rosé $^{3}$ \\ Published online: 22 March 2021 \\ (c) International Society of the Learning Sciences, Inc. 2021
}

\section{Introduction}

It is widely recognized that collaborative learning, particularly when organized around authentic inquiry problems, has many pedagogical benefits, including fostering deep learning, knowledge building and the development of twenty-first century skills such as collaboration, communication, creativity, and critical thinking. However, it is also well documented in the CSCL literature that productive collaboration does not happen automatically. CSCL research has produced guidelines for creating processes, conditions and tools, which are woven together to create the conditions that foster effective group interactions (Jeong \& Hmelo-Silver, 2016).

Productive collaborative learning and working is a multifaceted interaction process that integrates cognitive, motivational and emotional components as the core of collaboration (Borge et al., 2018; Ludvigsen, 2016). In theory, collaborative learning requires group members to be aware of and to coordinate their cognitive, metacognitive, motivational and emotional resources and processes (Hadwin et al., 2018). In practice, the foundation for successful CSCL rests on work focused on shared goals, communication in which knowledge and understanding are shared, and collaboration processes are jointly monitored (Zheng et al., 2019). These processes of regulation therefore form a layer around collaborative learning processes, working to ensure the success of the more basic processes. However, those regulation processes in turn depend upon support in order to be successful. Designs for computer support in its various forms comprise a final, outermost layer, strengthening regulation processes that support the basic processes of collaboration.

The cornerstone of pedagogical design for CSCL environments is to find a delicate balance between free-form collaboration and the structuring, scaffolding and orchestrating of

Carolyn Rosé

cp3a@andrew.cmu.edu

Nancy Law

nlaw@hku.hk

Sanna Järvelä

sanna.jarvela@oulu.fi

1 University of Hong Kong, Hong Kong, China

2 University of Oulu, Oulu, Finland

3 Carnegie Mellon University Language Technologies Institute and HCI Institute, Pittsburgh, PA, USA 
collaborative activities. Collaboration scripts are one common form of structuring support featured prominently within the development of the field, especially as it has progressed in the history of this journal since its inception in 2006. These are pedagogical designs for scaffolding that pre-structures the socio-cognitive processes of CSCL. CSCL scripts have been found to have some effect on domain learning but a more substantial effect on collaboration skills (Vogel et al., 2017). Orchestration refers to the management of teaching in the classroom context, particularly from the perspective of the coordination of activities such as individual work, small-group interaction and classroom discussions (Tchounikine, 2013). Because metacognition plays an important role in group learning, recent technologies have focused specifically on supporting metacognition in the context of CSCL. Typically, the support has been provided in the form of scripts or prompts that are used to facilitate learners' awareness of their metacognitive processes both at the individual and group levels (Janssen \& Bodemer, 2013).

More broadly, the field of CSCL produces research products in the form of empirically grounded guidelines for designing paradigms and supports in terms of a) pedagogical designs and b) technological tools for CSCL to overcome the interaction challenges and support critical CL processes. These can be enacted in advance to guide group activities, for example, during the groups' learning processes as a support for orienting to the productive collaboration or as a support for monitoring learning and interaction during the collaboration process.

The four papers in this issue explore the interplay of "layers" of design for supportive technologies that scaffold regulation of collaboration and learning. These regulation processes may influence more basic collaboration processes, and ultimately its outcomes. One layer in collaboration design focuses on the interlacing of collaborative learning interactions involving social units at different granularities, such as individual, intra-group, intergroup, and whole class interactions. We can refer to this as socio-organizational layering. Both Chen et al.'s and Zhang et al.'s papers focus on this type of layering. One layer out in collaboration design is the use of technological tools to scaffold the regulation and ultimately the collaboration processes. The Strauss et al. and Soni et al. papers belong to this second category.

\section{Regulation of collaborative processes: Exploring the layering of social units at different granularities}

The papers by Chen et al. and by Zhang et al. are similar not only in sharing a common focus on exploring layering of different social units in collaborative interactions, but also in sharing a common research context. Both studies were conducted within a teacher professional development course on technology-enhanced learning.

The first paper, by Wenli Chen, Jesmine Tan, and Zhongling Pi, is entitled "The Spiral Model of Collaborative Knowledge Improvement: An Exploratory Study of a Networked Collaborative Classroom”. This paper presents a study involving a class of 19 preservice teachers taking a course on using ICT in Chinese Language and Literature. A complete collaboration cycle in Chen et al.'s interaction design has five stages: individual ideation, intragroup sharing, inter-group critique, intragroup refinement and then completion of an individual assignment. The first four stages in each cycle are completed within one lesson (35 min), and the final stage of individual work was to be completed outside of lesson time. The entire course has 6 lessons. The basic rationale for this layered design is that it would 
provide different stimulus for student learning, and the outcome targeted was the uptake of constructs in the TPACK framework of technology-enhanced learning (TEL) in the preservice teachers' learning design. The author team concludes that this layered design is effective because there is a clear progression in terms of the quality of the TEL design according to the TPACK framework over the five phases of collaboration. The paper also analyzes the the quality of the TEL design for each group member in one of the groups during one lesson (i.e., one complete collaboration cycle) to demonstrate how the TEL expertise exhibited by different group members was expanded and became more similar between the first and the fifth phases in the collaboration cycle. The paper places a particular focus on the importance of phase 3-intergroup critique-in promoting knowledge improvement. The analysis illustrates through uptake analysis the impact of the connection between the refined ideas and the critiques received during phase 3 and later refinements in the appropriation of TPACK constructs in the learning designs.

The second paper, by Si Zhang, Juan Chen, Yun Wen, Hogxian Chen, Qianqian Gao, \& Qiyun Wang, is entitled "Capturing regulatory patterns in online collaborative learning: A network analytic approach". This study involved 45 preservice teachers from all disciplines on instructional design and courseware development. While Chen et al.'s pedagogical script for the collaboration design was grounded on a communication model, the research focus for Zhang et al.'s study was on exploring whether and how different layers of collaborative interaction engage different regulatory mechanisms involved in collaboration. Zhang et al.'s study has a simpler collaboration cycle, each comprising three stages: intra-group discussion, inter-group assessment, and intra-group refinement. Two complete cycles of collaboration were implemented over the 8 weeks of a course's duration, with each stage taking place over one week.

A detailed coding scheme was developed to analyze the pre-service teachers' regulation behavior as reflected in their online discussions. Each group's achievement in terms of the quality of the learning design artefacts produced was assessed by two subject matter experts. The research team selected a high performing group and a low performing group to compare the similarities and differences in the students' regulation behavior across contexts. Important differences in regulatory behavior were identified between the high performing and low performing groups. By employing Epistemic Network Analysis (ENA), Zhang et al. found that the high performing group engages more in planning and shows high correlation between monitoring and evaluation at each stage of the collaboration, whereas the low performing group demonstrated higher correlation between monitoring and regulation. Furthermore, the high performing group invested more effort in content monitoring.

As CSCL gains popularity in adoption, there is an increasing need to develop more refined pedagogical designs to scaffold the collaboration process. Both Chen et al.'s and Zhang et al.'s papers show an interest in the layering of collaborative interactions involving intra-group and inter-group interactions. Their findings also suggest that such pedagogical scripting in the form of cycles of collaboration makes a difference.

\section{Layering of technology support for regulation}

The final two papers of the March edition of the journal report about research that explores the properties of alternative supportive technologies, for example, Group Awareness Tools (GATs) and Adaptive Collaboration Prompts (ACPs) designed to foster regulation towards processes of equal participation as the Strauss and Rummel 
paper. On the other hand, the Soni and colleagues' paper investigates the multilayered nature of the interactions that can be afforded by emerging technologies such as multitouch tabletop technologies.

In particular, the third article in this issue, by Sebastian Strauss and Nikol Rummel, is entitled "Promoting regulation of equal participation in online collaboration by combining a group awareness tool and adaptive prompts. But does it even matter?" Strauss and Rummel's paper explores whether the use of Group Awareness Tools (GATs) and Adaptive Collaboration Prompts (ACPs) will bring more equal participation. Achieving more equal participation also involves regulation from the learners, including self-regulation, co-regulation and socially shared regulation. The study features an experimental design to compare three conditions: GAT use only, GAT and ACP, and a control condition. The findings do not show statistically significant differences, even though the availability of GAT appears to be associated with more equal participation. The students did not find the ACP to be useful in prompting more regulatory behavior. What appears to emerge from the findings is that students find it difficult to take socially shared regulatory action even if they are aware of the unequal participation. Perhaps an insight from this study is the need for pedagogical design to promote socially shared regulation. Just as productive collaborative learning does not automatically take place because students are arranged for the purpose of engaging in a collaborative task, regulatory behavior needed for productive collaboration does not spontaneously emerge when students are aware of unequal participation. What counts as an appropriate indicator of participation is another issue that this study exposes. Word counts have been used as a default measure of participation. However, this is a very blunt measure and can hardly be equated to the quality of the contributions made by participants.

The final paper of the edition, by Nikita Nandish Soni, Alice Darrow, Annie Luc, Schuyler Gleaves, Carrie Schuman, Hannah Neff, Peter Chang, Brittani Kirkland, Jeremy Alexandre, Amanda Morales, Kathryn A. Stofer, and Lisa Anthony, is entitled "Affording Embodied Cognition through Touchscreen and Above-the-Surface Gestures During Collaborative Tabletop Science Learning". While GATs and ACPs are technological tools designed to circumvent in some ways the limitations of Internet-mediated collaboration, large touchscreen devices such as multitouch tabletops support and mediate face-to-face interactions and collaboration. Soni et al.'s paper shows the multilayered nature of the interactions that can be afforded by multitouch tabletop technologies. At the basic level, users can interact with a visual representation of a computational model of a particular domain, in this case earth science data. Different users may focus on different aspects of the information presented and make sense of the visual representations in different ways. Studies of embodied cognition have shown that gestures are important means of communicating conceptual understanding (Danish et al., 2020). This type of embodied cognition is also observed in the communicative behavior of collaborative groups interacting around a multitouch tabletop in Soni et al.'s study. However, what has previously been less studied is the use of gestures in regulating collaborative interactions. Soni et al. address this gap. In particular, they have identified three types of gestures to achieve three co-regulatory.goals: directing group attention to certain parts of the tabletop interface (to achieve shared attention), directing group attention to and sharing of personal meaning making outcomes, and gaining collaborative support from the group through direct interactions with the tabletop and above the surface gestures. These gestures play an important role in regulating the collaborative interactions beyond the use of linguistic cues (Dindar et al., 2020). 


\section{Bringing the layers together}

The work reported in this edition breaks new ground and also prompts new questions. For example, the differences in terms of pedagogical and research design between the Chen et al. and Zhang et al. papers highlight several important issues that warrant further exploration from theoretical and implementation perspectives. First of all, is there an optimum duration for each stage of collaboration for a specific social interaction setting? What is the theoretical basis for the duration and sequencing of the layering? Why should intra-group and inter-group interactions bring about different collaborative learning outcomes? Is there a theoretical model that can explain such differences?

From the technology side, we move on from the Strauss and Rummel paper and Soni et al. paper to ask if there are particular interface and interaction designs, either in terms of GATs or of multitouch tabletops that would positively or negatively influence embodied co-regulatory interactions? What are the fundamental differences in opportunity between these approaches, which appear so different at the surface. The differences in interface design between GATs with prompts and that of multitouch tabletops may each support coregulatory behaviors that ultimately foster cognition. Both papers point to specific open questions and next steps for the work.

\section{References}

Borge, M., Ong, Y. S., \& Rosé, C. P. (2018). Learning to monitor and regulate collective thinking processes. International Journal of Computer-Supported Collaborative Learning, 13, 61-92.

Danish, J. A., Enyedy, N., Saleh, A., \& Humburg, M. (2020). Learning in embodied activity framework: A sociocultural framework for embodied cognition. International Journal of Computer-Supported Collaborative Learning, 15, 49-87.

Dindar, M., Järvelä, S., Ahola, S., Huang, X., \& Zhao, G. (2020). Leaders and followers identified by emotional mimicry during collaborative learning: A facial emotions recognition study. IEEE Transactions on Affective Computing. https://doi.org/10.1109/TAFFC.2020.3003243.

Hadwin, A. F., Järvelä, S., \& Miller, M. (2018). Self-regulation, co-regulation, and shared regulation in collaborative learning environments. In D. H. Schunk \& J. A. Greene (Eds.), Handbook of self-regulation of learning and performance (2nd ed., pp. 83-106). Routledge.

Janssen, J., \& Bodemer, D. (2013). Coordinated computer-supported collaborative learning: Awareness and awareness tools. Educational Psychologist, 48, 40-55.

Jeong, H., \& Hmelo-Silver, C. E. (2016). Seven affordances of computer-supported collaborative learning: How to support collaborative learning? How can technologies help? Educational Psychologist, 51, 247-265.

Ludvigsen, S. (2016). CSCL: connecting the social, emotional and cognitive dimensions. International Journal of Computer-Supported Collaborative Learning, 11, 115-121.

Tchounikine, P. (2013). Clarifying design for orchestration: Orchestration and orchestrable technology, scripting and conducting. Computers \& Education, 69, 500-503.

Vogel, F., Wecker, C., Kollar, I., \& Fischer, F. (2017). Socio-cognitive scaffolding with computer-supported collaboration scripts: A meta-analysis. Educational Psychology Review, 29, 477-511.

Zheng, J., Xing, W., \& Zhu, G. (2019). Examining sequential patterns of self- and socially shared regulation of STEM learning in a CSCL environment. Computers \& Education, 136, 34-48.

Publisher's note Springer Nature remains neutral with regard to jurisdictional claims in published maps and institutional affiliations. 